\title{
Numerical Simulations of Laser-Driven Cratering Experiments into Porous Graphite
}

\author{
Bertrand Aubert ${ }^{1, *}$, David Hebert $^{1}$, Jean-Luc Rullier ${ }^{1}$, Jean-Marc Chevalier ${ }^{1}$, Laurent Berthe ${ }^{2}$, Erik Brambrink $^{3}$, Emilien \\ Lescoute $^{4}$, Laurent Videau ${ }^{4}$, Jean-Eloi Franzkowiak ${ }^{4}$, Benjamin Jodar ${ }^{5}$, Didier Loison ${ }^{5}$, and Mariette Nivard ${ }^{5}$ \\ ${ }^{1}$ CEA CESTA, 15 Avenue des Sablières, CS60001, 33116 Le Barp Cedex, France \\ ${ }^{2}$ Laboratoire PIMM, UPR80006 CNRS-ENSAM, 151 Boulevard de l'Hopital, 75013 Paris, France \\ ${ }^{3}$ Laboratoire pour l'Utilisation des Lasers Intenses, UMR7605 CNRS-CEA-Ecole Polytechnique-Université Paris VI, 91128 \\ Palaiseau, France \\ ${ }^{4}$ CEA DIF, Bruyères-le-Châtel, 91297 Arpajon Cedex, France \\ ${ }^{5}$ Institut de Physique de Rennes, CNRS-Université de Rennes 1, 35042 Rennes Cedex, France
}

\begin{abstract}
We present the results of an experimental campaign conducted on the LULI2000 laser facility. Semiinfinite targets of a commercial grade of porous graphite were submitted to high-power laser irradiation in order to generate craters. A $15 \mathrm{~ns}$ pulse duration was used along with a focal spot diameter of $900 \mu \mathrm{m}$ to deliver energies up to $750 \mathrm{~J}$. Numerical simulations of these shots have been performed following a specific methodology which can be divided in three steps. Firstly, the mechanical loading induced by the laser is calibrated by simulating the same shot on a thin aluminum target of which free surface velocity is measured by PDV and line-VISAR. Secondly, the same shot is performed on a thin graphite target to validate the material model of graphite. Thirdly, the craterization shot on semi-infinite target is simulated. Numerical results are compared to experimental measurements of craters obtained using an interferometric profilometer.
\end{abstract}

\section{Introduction}

The cratering process in brittle materials is a major concern for the aerospace industry which has to design satellites and spacecrafts able to withstand impacts of meteoroids or debris at several kilometers per second. Complementary to laboratory launchers, high-power laser facilities could allow to investigate this process $[1,2]$. Indeed, the interaction of the laser with target material generates a plasma whose expansion creates a shock wave that may lead to the formation of a crater.

In this paper, we present laser-driven cratering experiments performed on the LULI2000 laser facility and the corresponding simulations. Such simulations require a preliminary study on thin targets to determine the mechanical loading induced by the laser on the target. With thin targets, it is possible to record the velocity of the back surface. Comparing these measurements with numerical results, we can access to the laser loading. The material chosen for this study is a porous graphite called EDM3 but, in a first step, we perfom a shot on a thin aluminum target because this well-known material does not raise any problem of modeling. In a second step, the same shot is performed on a thin EDM3 target to validate our model of this material. Finally, we simulate the craterization shot on semi-infinite EDM3 target. We note that EDM3 has already been studied under hypervelocity impacts $[3,4]$ and laser-driven shocks $[5,6]$.

\footnotetext{
*e-mail: bertrand.aubert@ cea.fr
}

Section 2 is devoted to the presentation of experimental set-up and main results. Simulations are investigated in Section 3 where a specific case is completely studied.

\section{Experiments}

\subsection{Experimental set-up}

The LULI2000 laser facility is a Nd-YAG high-power laser located at the Ecole Polytechnique (Palaiseau, France). It can deliver energies up to $800 \mathrm{~J}$ at the wavelength of $1064 \mathrm{~nm}$ with tunable pulse duration. All the shots presented in this paper were performed using square temporal profiles of $15 \mathrm{~ns}$ which were measured at each shot. The energy has been measured with a calorimeter at each shot too. A phase plate has been used to shape the focal spot before its entry in the vacuum chamber containing the target. The spot has been recorded with a CCD camera placed at the target position. From this measurement, shown in Figure 1, we estimate a focal spot diameter of about $900 \mu \mathrm{m}$. Repetition of this procedure showed that the spatial distribution was stable during all the campaign.

The two materials shot during this campaign were aluminum 6061-T6 and EDM3. This later is a porous graphite commercialized by POCO which can be considered as macroscopically isotropic. It has a density of $1754 \mathrm{~kg} \mathrm{~m}^{-3}$ and a porosity of $22 \%$. Its main mechanical characteristics can be found in [4]. Three types of targets have been used: 


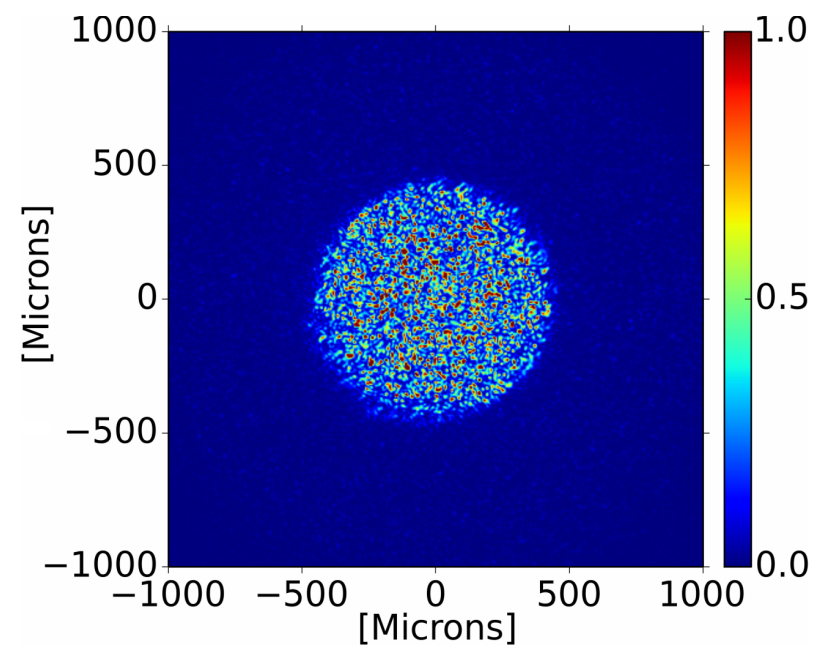

Fig. 1. Spatial distribution of energy measured by a CCD camera at the target position.

- thin targets made of $197 \pm 2 \mu \mathrm{m}$ of aluminum 6061-T6 bonded to a $2 \mathrm{~mm} \mathrm{LiF}$ window;

- thin targets made of $191 \pm 2 \mu \mathrm{m}$ of EDM3 bonded to a $2 \mathrm{~mm} \mathrm{LiF}$ window;

- semi-infinite targets made of $8 \mathrm{~mm}$ of EDM3.

$\mathrm{LiF}$ windows are bonded to the back face of thin targets and the velocity is measured through these transparent windows. In this way, we measure the particle velocity at the interface between the target and the window. This velocity is easier to measure than the free surface velocity which is approximatively twice higher. Moreover, the shock impedance of $\mathrm{LiF}$ is almost equal to that of aluminum. Consequently, the use of a $\mathrm{LiF}$ window allows to observe the release which follows the shock without being interrupted by shock reflection at the $\mathrm{Al} / \mathrm{LiF}$ interface. The glue thickness between the target and the window will be neglected in the rest of the paper.

To measure the velocity on thin targets, we used two complementary diagnostics: a line-VISAR [7] and a multipoint PDV system [8]. Our line-VISAR measures the velocity on a line $5 \mathrm{~mm}$ long during about $20 \mathrm{~ns}$ with a temporal resolution less than a nanosecond at a wavelength of $532 \mathrm{~nm}$. It gives us precisely the arrival time of the shock and the velocity at this moment but the measurement duration is too short to observe the whole shock. This is why we also used a multipoint PDV system which measures the velocity during a few microseconds with a temporal resolution of a few nanoseconds at a wavelength of $1550 \mathrm{~nm}$. Our PDV system has the particularity to contain eight punctual probes spaced $250 \mu \mathrm{m}$ apart so, like the line-VISAR, it gives us an information on the loading at the center of the focal spot but also around it. Such diagnostics are very interesting to study the cratering process because it is clearly a two-dimensional mechanism for which radial effects are significant. Even if the wavelengths of these two diagnostics were different, they were not compatible because of space limitations.

\subsection{Results}

Three craters have been generated in semi-infinite EDM3 targets at $83 \mathrm{~J}, 445 \mathrm{~J}$ and $662 \mathrm{~J}$. All these craters have been measured with an interferometric profilometer. It provides a 3D map of each crater from which we measure the maximum depth $d$, the diameter $\phi$ and the volume $V$ and we extract a $2 \mathrm{D}$ profile crossing the crater center. If the definitions of $d$ and $V$ are not ambiguous, it is more complicated for $\phi$. Indeed, the crater is always composed of a central volume surrounded by a ring. This specific shape is visible on the profile shown in Figure 2. Sometimes the outer ring is not formed all around the crater so it is difficult to precisely measure a diameter. It is for this reason, that we prefer distinguish two diameters: the diameter of the central volume denoted $\phi_{1}$ and the diameter of the outer ring denoted $\phi_{2}$.

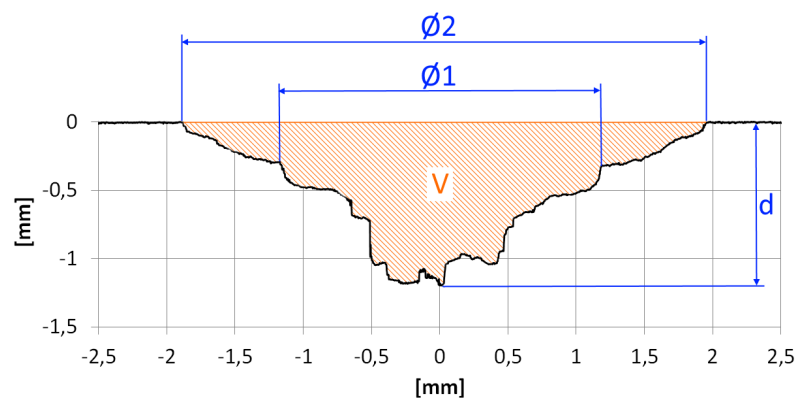

Fig. 2. Definition of $V, d, \phi_{1}$ and $\phi_{2}$ on the $445 \mathrm{~J}$ crater profile.

Figure 3 shows the evolution of crater dimensions as a function of the laser energy. This energy is the one that has been measured by the calorimeter and not the one that has been adjusted by numerical simulations (see 3.2). It has already been observed for this material that the crater volume increases linearly with the laser energy [5] but the evolution does not seem linear in the present case (Figures 3.a). It can be explained by the fact that the outer ring of the $445 \mathrm{~J}$ crater is complete whereas that of the $662 \mathrm{~J}$ crater is incomplete. The ring seems to be due to a crack which grows radially all around the crater and ejects matter when it becomes sufficiently significant. Crack propagation strongly depends on the defect distribution in the material and the spatial distribution of the energy in the laser spot so three shots are not enough to clearly discriminate a trend concerning V. Contrariwise, the quantities $d, \phi_{1}$ and $\phi_{2}$ are not problematic because their definitions remain valid if the outer ring is incomplete. They are proportional to the laser energy at the power $1 / 3$ (Figures 3.b and 3.c). These results are consistent with previous observations at lower intensities [5].

We simulated step by step these three shots, including the preliminary characterization shots on thin targets. For the sake of brevity, we will only present in Section 3 the case of the $662 \mathrm{~J}$ crater. Table 1 lists all the characterization shots relative to this laser configuration. Thanks to shots 1 and 2 which are complementary, we will be able to calibrate the loading. These two shots can be considered as identical because their energies differ by less than 

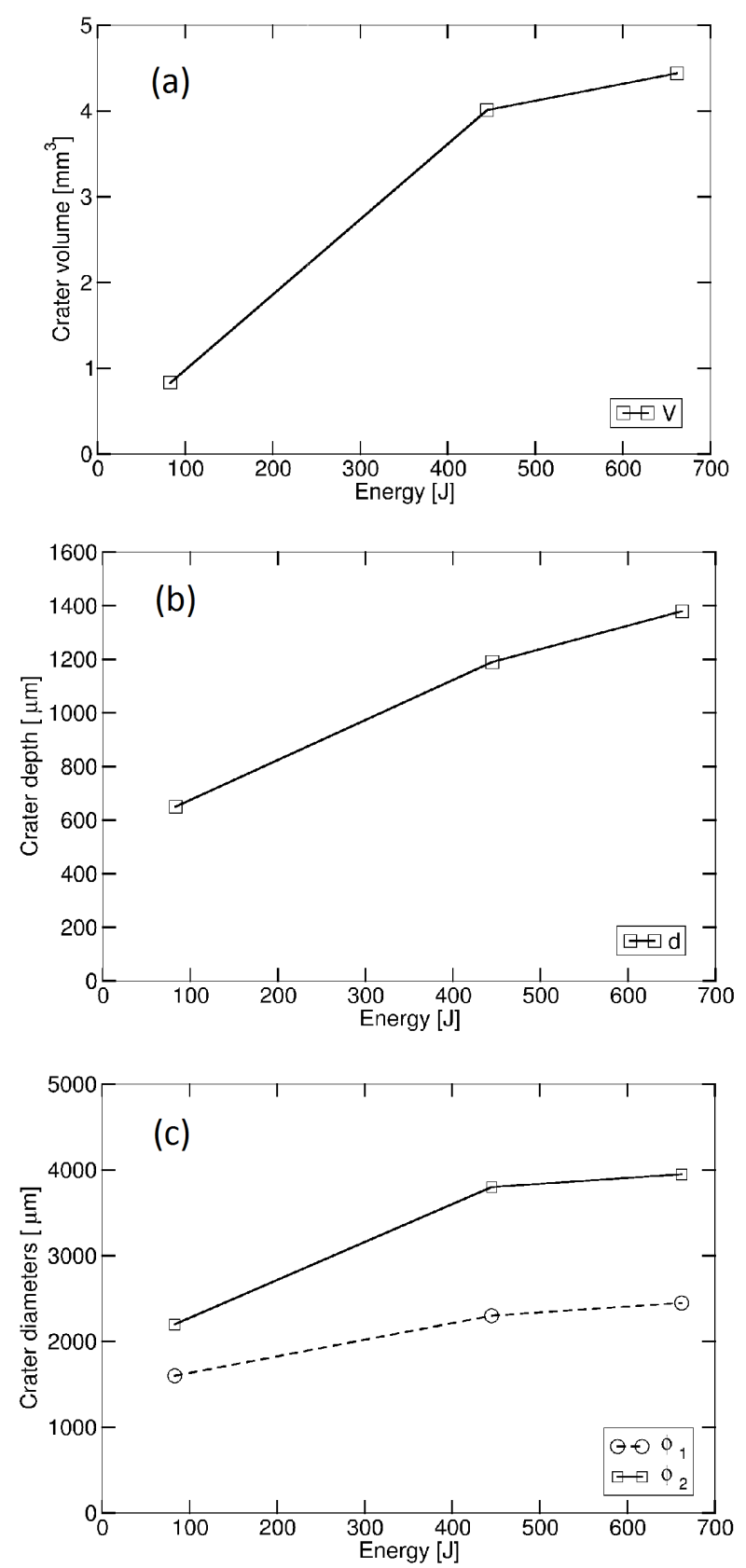

Fig. 3. Evolution of $V(\mathrm{a}), d(\mathrm{~b}), \phi_{1}$ and $\phi_{2}$ (c) as a function of laser energy.

$4 \%$ and their temporal profiles are similar. Shot 3 will be useful to validate our modelling of the EDM3 before to simulate the craterization shot.

Table 1. List of characterization shots relative to the $662 \mathrm{~J}$ craterization shot

\begin{tabular}{llll}
\hline Shot & Energy & Target & Diagnostic \\
\hline 1 & $742 \mathrm{~J}$ & $\mathrm{Al}(197 \mu \mathrm{m})+\mathrm{LiF}(2 \mathrm{~mm})$ & PDV \\
2 & $717 \mathrm{~J}$ & $\mathrm{Al}(197 \mu \mathrm{m})+\mathrm{LiF}(2 \mathrm{~mm})$ & VISAR \\
3 & $717 \mathrm{~J}$ & EDM3 $(191 \mu \mathrm{m})+\mathrm{LiF}(2 \mathrm{~mm})$ & PDV \\
\hline
\end{tabular}

\section{Simulations}

\subsection{Codes and models}

To treat the laser/matter interaction we used the Lagrangian mono-dimensional ESTHER code. The shock propagation and the mechanical response of the material were performed with the HESIONE Eulerian hydrocode in 2D-axisymmetrical geometry.

The behaviour of aluminum 6061-T6 is described with the equation of state (EOS) SESAME 3720 and the SCG model [9]. The set of parameters used for this model is presented in [10]. In the same way, the EOS SESAME 7270 and the SCG model are used for the LiF. The behaviour of EDM3 is described with the POREQST model [11]. This model takes into account the pores closure and the pores reopening in porous materials. It has been calibrated for our EDM3 on a large range of experiments. All the selected parameters are summarized in [3].

\subsection{Calibration of the laser loading on thin targets}

From Figure 1, we extract the radial profile $f(r)$ shown in Figure 4. Beyond a radius of about $1250 \mu \mathrm{m}$ the energy measured by the CCD camera is too low compared to the measurement noise so we extrapolate the profile using an exponential decrease.

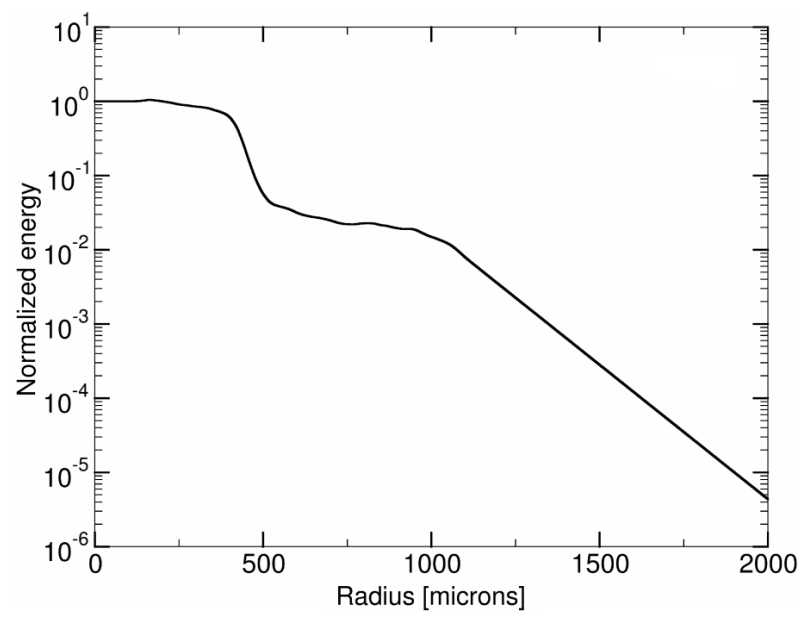

Fig. 4. Normalized radial profile of energy in logarithmic scale.

Knowing $f(r)$ and the laser energy, we can determine the fluence at the center of the spot. This fluence and the measured temporal profile of the shot 1 (Figure 5) are used as input data for an ESTHER simulation. As output, we got the ablation pressure $P(r=0, t)$. To obtain the complete pressure field $P(r, t)$, we use the radial profile of energy $f(r)$ and the Gr?n law [12] which claims that the ablation pressure is proportional to the laser intensity at the power 0.8:

$$
P(r, t)=P(r=0, t) f(r)^{0.8}
$$

This pressure field is then applied on the target surface as boundary condition in the HESIONE code. From this simulation, we can extract the velocity at the $\mathrm{Al} / \mathrm{LiF}$ interface for different radii and compare these results to PDV and 
VISAR measurements. Without any adjustments, simulation overestimates experimental measurements by about $20 \%$. We know that a part of the laser energy is lost in the last optics of the laser chain (lens, window and phase plate) which are placed after the measurement point of the energy. Thanks to our simulations, we estimate this loss at $20 \%$. In the rest of this paper, all the numerical results which are presented take this loss into account.



Fig. 5. Normalized temporal profile of energy for the shot 1 .

Figure 6 shows numerical results for the shot 1 (bold straight lines). Velocities at $\mathrm{r}=0 \mu \mathrm{m}$ and $\mathrm{r}=750 \mu \mathrm{m}$ are compared with corresponding PDV measurements (dotted lines). All other PDV measurements are unusable for this shot. Because of its poor temporal resolution, the PDV system was not able to precisely measure the peak of velocity for this shot. Indeed, the velocity increases too rapidly until a very high level to be captured by PDV. But, once the peak passed, PDV measures with reliability the release behind the shock. The velocity measured with line-VISAR at $\mathrm{r}=0 \mu \mathrm{m}$ for the shot 2 (thin straight line) is also plotted in Figure 6. This velocity is extracted from raw measurement shown in Figure 7. The high deformation of the $\mathrm{Al} / \mathrm{LiF}$ interface on the shock edges implies a lost of reflectivity. The fringe shift becomes unusable and the velocity cannot be measured in this area. Globally, our simulation well reproduces the arrival time of the shock, the velocity level and the shape of the release, even at $\mathrm{r}=750 \mu \mathrm{m}$.

Our modeling of the loading is validated so we simulate the shot 3 on thin EDM3 target with the same procedure. Only the PDV measurement at $\mathrm{r}=0 \mu \mathrm{m}$ is usable for this shot. This time, the peak of velocity has been measured. It is compared with the corresponding numerical result in Figure 8. The shape of the release is well reproduced and the velocity level too. The velocity jump observed at about $50 \mathrm{~ns}$ is due to a shock reflection which occurs at the interface between EDM3 and LiF because their shock impedances are different. We cannot use the PDV measurement to discuss the arrival time of the shock because its temporal resolution is of a few nanoseconds.



Fig. 6. Comparison of numerical and experimental results for shots 1 and 2 on thin aluminum targets.

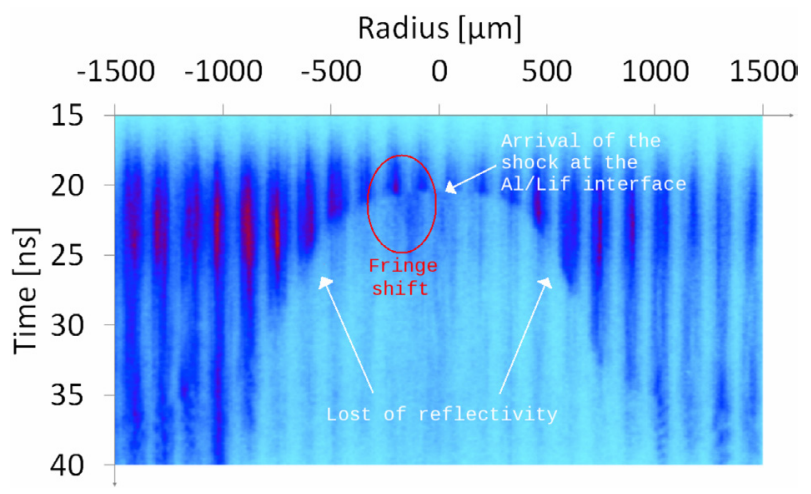

Fig. 7. Line-VISAR measurement for shot 2.

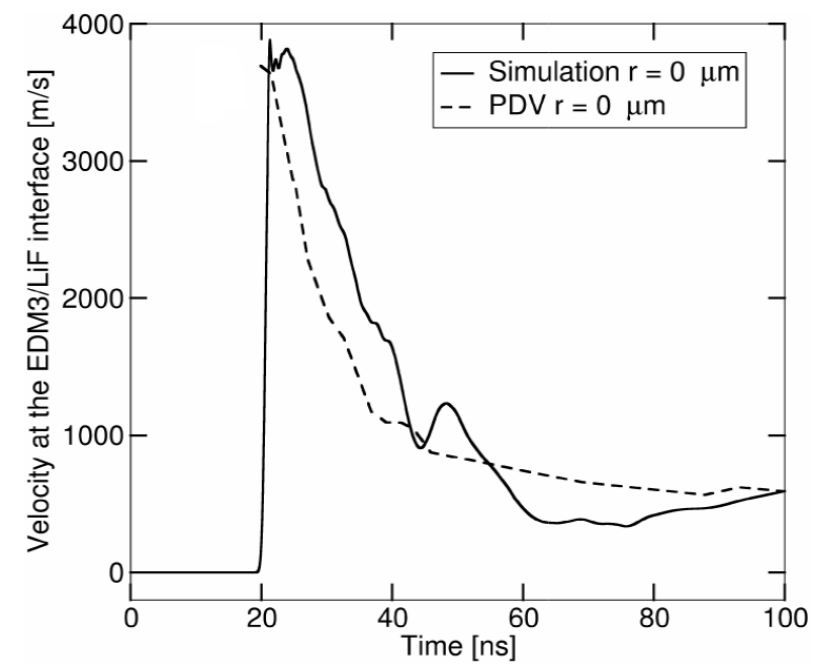

Fig. 8. Comparison of numerical and experimental results for shots 3 on thin EDM3 target. 


\subsection{Simulation of a craterization shot on semi-infinite target}

In this part, we present the simulation of the $662 \mathrm{~J}$ craterization shot on semi-infinite target of EDM3. The result of this simulation is compared with a cut of the experimental crater on Figure 9. The red area on the simulation corresponds the area where the damage criteria of our model has been reached. Such material is completely damaged and no longer has any tensile strength. The experimental profile should be contained in this area.

As we can see, our simulation well reproduces the central volume and therefore $d$ and $\phi_{1}$. But, the outer ring is not reproduced numerically so $V$ and $\phi_{2}$ are not provided by our simulation. However, we distinguish a beginning of crack on the crater edges. It is this crack which could be at the origin of the formation of the outer rings.



Fig. 9. Comparison between experimental profile (black line) and simulation for the $662 \mathrm{~J}$ shot.

\section{Conclusion}

We performed three laser shots in semi-infinite EDM3 targets at very high intensities. Resulting craters have been measured and their particular shape has been noticed. They are always formed of a central volume surrounded by an outer ring. Numerical simulation of one of these shots has been fully described. Such a simulation requires a preliminary study on thin targets to calibrate the loading. This step has been validated by the good agreement observed between numerical simulations and experimental PDV and VISAR measurements. Finally, the complete simulation of the craterization shot well reproduces the central volume of the crater but not the outer ring.

\section{References}

1. P. E. Nebolsine. Laser simulation of hypervelocity impact. Proceedings of the AIAA 14th ASM (1976)
2. A. N. Pirri. Theory for laser simulation of hypervelocity impact. Physics of Fluids 20, 221-228 (1977)

3. D. Hebert, G. Seisson, J.-L. Rullier, I. Bertron, L. Hallo, J.-M. Chevalier, C. Thessieux, F. Guillet, M. Boustie, and L. Berthe. Hypervelocity impacts into porous graphite: experiments and simulations. Philosophical Transactions of the Royal Society A 375, 2085 (2016)

4. G. Seisson, D. Hebert, I. Bertron, J.-M. Chevalier, L. Hallo, E. Lescoute, L. Videau, P. Combis, F. Guillet, M. Boustie, and L. Berthe. Dynamic cratering of graphite: experimental results and simulations. International Journal of Impact Engineering 63, 18-28 (2014)

5. B. Aubert, D. Hebert, J.-L. Rullier, I. Bertron, F. Malaise, L. Videau, E. Lescoute, and L. Berthe. Laser driven cratering of porous graphite: experiments and simulations. Proceedings of the 22nd DYMAT Technical Meeting (2016)

6. G. Seisson, G. Prudhomme, P.-A. Frugier, D. Hebert, E. Lescoute, A. Sollier, L. Videau, P. Mercier, M. Boustie, and L. Berthe. Dynamic fragmentation of graphite under laser driven shocks: Identification of four damage regimes. International Journal of Impact Engineering 91, 68-79 (2016)

7. L. M. Barker, and R. E. Hollenbach. Laser interferometer for measuring high velocities of any reflecting surface. Journal of Applied Physics 43, 4669-4675 (1972)

8. O. T. Strand, D. R. Goosman, C. Martinez, and T. L. Whitworth. Compact system for high speed velocimetry using heterodyne techniques. Review of Scientific Instruments 77083108 (2006)

9. D. J. Steinberg, S. G. Cochran, and M. W. Guinan. A constitutive model for metals applicable at high strain rate. Journal of Applied Physics. 51, 545-547 (1981)

10. B. Aubert, D. Hebert, J.-L. Rullier, E. Lescoute, L. Videau, and L. Berthe. 2D characterization of the pressure generated by an intense laser pulse on an aluminum target. Proceedings of the 22th APS SCCM Conference (2017)

11. L. Seaman, R. E. Tokheim, and D. R. Curran. Computational representation of constitutive relations for porous material. SRI Technical Report DNA-3412F (1974)

12. J. Grun, R. Decoste, B. H. Ripin, and J. Gardner. Characteristics of ablation plasma from planar, laser driven targets. Applied Physics Letters 39, 545-547 (1981) 
